\title{
Alergias Alimentares e Não Alimentares Entre Pacientes Assistidos em Ambulatório de Alergia e Imunologia
}

\author{
Food and Non-Food Allergies Among Outpatient Patients \\ of Allergy and Immunology
}
Alergias Alimentarias y no Alimentarias entre Pacientes Asistidos en Ambulatorio de Alergia e Inmunología

Raquel Dantas de Andrade ${ }^{1}$

Andressa Carolina de Oliveira Mundim ${ }^{2}$

Kathiane Pereira de Jesus ${ }^{3}$

Jairo Calado Cavalcante ${ }^{4}$

Iramirton Figuerêdo Moreira ${ }^{5}$

\section{Resumo}

Objetivo: avaliar o perfil dos pacientes registrados no ambulatório de Alergia e Imunologia do Hospital Universitário de AL. Métodos: foi realizado um estudo transversal analítico com dados secundários, através da análise do livro de registro de diagnósticos do ambulatório de Alergia e Imunologia do Hospital Universitário AL, de pacientes atendidos entre outubro de 2016 e setembro de 2017. O banco de dados foi montado no programa Microsoft Excel 2017 e analisado pelo programa EpiInfo 7.2.2.6, para a análise estatística foi aplicado o teste de Quiquadrado no nível de confiança de 95\%, para a análise da relação entre idade e alergia, os pacientes foram separados em 3 faixas etárias, de zero a menos de 10 anos, de 10 a 18 anos e maiores de 18

${ }^{1}$ Discente do curso de graduação em Medicina pela Universidade Federal de Alagoas (UFAL). Autora correspondente: Campus A. C. Simões. Av. Lourival Melo Mota, s/n. Tabuleiro dos Martins. 57072-900. Maceió, Al, Brasil. E-mail: raquel.andrade@outlook.com

${ }^{2}$ Discente do curso de graduação em Medicina pela Universidade Federal de Alagoas (UFAL)

${ }^{3}$ Professor de Bioestatística, especialista em epidemiologia, da Faculdade de Medicina da Universidade Federal de Alagoas (FAMED/UFAL).

${ }^{4}$ Médico Especialista em Alergia e Imunologia, Mestre, Médico da EBSERH/HUPAA e Professor Adjunto da Faculdade de Medicina da Universidade Federal de Alagoas (FAMED/UFAL).

Recebido: Abr./2018 - Aceito: Ago./2018. 
anos.. Resultados: dos 205 pacientes registrados que foram incluídos na amostra, 95 (46,34\%) eram do sexo feminino. A idade média foi de 16,5 anos, com desvio padrão de 17,3. A alergia alimentar foi a mais prevalente, encontrada em 98 (47,8\%) indivíduos, sendo a proteína do leite de vaca o principal desencadeante. Foi encontrada significância estatística na associação entre alergia e faixa etária, tendo-se maior prevalência de alergia na faixa etária entre 0 a 10 anos. As reações a fármacos foram a segunda causa mais prevalente, obtendo um total de 81 (39,5\%) pacientes, composta principalmente pelos anti-inflamatórios não esteroidais. As imunodeficiências primárias foram identificadas em 11 $(5,4 \%)$ da amostra. Conclusão: O perfil dos pacientes registrados no ambulatório de alergia e imunologia é semelhante com os descritos da literatura.

\section{Descritores: Hipersensibilidade}

Alimentar; Hipersensibilidade;

Imunidade.

\section{Abstract}

Objective: To evaluate the profile of patients enrolled in the Allergy and Immunology outpatient clinic of the
University Hospital of AL. Methods: An analytical cross-sectional study with secondary data was carried out through the registry book of diagnoses of the Allergy and Immunology outpatient clinic of the University Hospital of Al, with the patients that attended between October 2016 and September 2017. The database was mounted in the Microsoft Excel 2017 program and analyzed by the EpiInfo 7.2.2.6 program, for the statistical analysis, the Chi-square test was applied at the 95\% confidence level, to analyze the relationship between age and allergy, patients were separated into 3 age groups, from zero to less than 10 years, from 10 to 18 years and over 18 years. Results: From 205 patients enrolled and included in the sample, 95 (46.34\%) were female. The mean age was 16.5 years, with a standard deviation of 17.3. Food allergy was the most prevalent found in 98 (47.8\%) individuals and the main triggering agent among food allergies was cow's milk protein. Statistical significance was found in the association between allergy and age group, with a higher prevalence of allergy in the age group between 0 and 10 years. Drug reactions were the second most prevalent cause, obtaining a total of $81 \quad(39.5 \%)$ patients, composed mainly of nonsteroidal 
antiinflammatory drugs. Primary immunodeficiencies were identified in $11(5.4 \%)$ of the sample. Conclusion: The profile of patients enrolled in allergy and immunology clinic is similar to those described in literature.

\section{Descriptors: Food Hypersensitivity;} Hypersensitivity; Immunity.

\section{Resumen}

Objetivo: evaluar el perfil de los pacientes registrados en el ambulatorio de Alergia e Inmunología del Hospital Universitario de AL. Se realizó un estudio transversal analitico con datos secundarios a través del análisis del libro de registro de diagnósticos del ambulatorio de Alergia e Inmunología del Hospital Universitario de AL, los pacientes atendidos entre octubre de 2016 y septiembre de 2017. La base de datos fue montada en el programa Microsoft Excel 2017 y analizado por el programa EpiInfo 7.2.2.6, para el análisis de la relación entre edad y alergia, los pacientes se separaron en 3 grupos de edad, de cero a menos de 10 años, de 10 a 18 años, y mayores de 18 años. Resultados: De los 205 pacientes registrados y que fueron incluidos en la muestra, el 95 (46,34\%) eran del sexo femenino y el $110 \quad(53,33 \%)$ del masculino. La edad media fue de 16,4 años, con desviación estándar de 17,3. La alergia alimentaria fue la más prevalente, encontrada en 98 (47,8\%), siendo el proteína de la leche de vaca el principal agente desencadenante. Se encontró una significancia estadística en la asociación entre alergia y grupo de edad, teniendo una mayor prevalencia de alergia en el grupo de edad entre 0 a 10 años. Las reacciones a fármacos fueron la segunda causa más prevalente obteniendo un total de 81 (39,5\%) pacientes, compuesta principalmente por los antiinflamatorios no esteroidales. Las inmunodeficiencias primarias fueron identificadas en el $11(5,4 \%)$ de la muestra. Conclusión: El perfil de los pacientes registrados en el ambulatorio de alergia e inmunología es similar con los descritos en la literatura.

Descriptores: Hipersensibilidad a los Alimentos; Hipersensibilidad; Inmunidad.

\section{Introdução}

A prevalência das doenças alérgicas está aumentando em todo o mundo, especialmente em crianças e adultos jovens $^{(1)}$. Estima-se que cerca de 200 a 250 milhões de pessoas no mundo 
apresentam alergia alimentar e um décimo da população sofra de alergia a fármacos, além do que, frequentemente as doenças alérgicas ocorrem sobrepostas em um mesmo indivíduo. Mudanças ambientais, dieta, estilo de vida e comportamento individual têm papel fundamental neste fenômeno, propiciando a população a se tornar sensibilizada ${ }^{(1)}$.

As alergias alimentares e medicamentosas são reações de hipersensibilidade provocada por uma resposta imunomediada que ocorre de forma reprodutiva na exposição a um determinado alérgeno ${ }^{(2,3)}$. Os alimentos mais relacionados com alergia em crianças são leite e ovo e as manifestações clínicas variam de reações cutâneas localizadas até uma condição de risco á vida, como a anafilaxia $^{(4)}$. As reações adversas a medicamentos também têm aumentado e são uma consequência do benefício da terapia medicamentosa atual. Cada droga é associada à um leque de reações adversas, que vão de afecções leves até condições raras e graves ${ }^{(1)}$.

As imunodeficiências primárias (IDPs) consistem em desordens imunológicas heterogêneas, capazes de acometer a imunidade adaptativa e inata, tendo alta morbidade ${ }^{(5)}$. Na maioria dos casos são anormalidades genéticas que se manifestam nos primeiros anos de vida, através de longos e reincidentes processos infecciosos ${ }^{(5)}$. Embora sejam consideradas doenças de incidência rara, estima-se que as IDPs ocorram em mais de 1 a cada 2.000 nascimentos ${ }^{(6)}$. Dentre elas, se destacam: a imunodeficiência combinada grave, a gamaglobulinemia ligada ao $\mathrm{X}$, síndrome hiper-IgM ligada ao $\mathrm{X}$, ataxiatelangectasia, imunodeficiência variável comum e síndrome de WiskottAldrich $^{(5)}$.

Ao apresentar o perfil da população atendida, o estudo identifica e reporta o padrão e a frequência de eventos relacionados à saúde, demonstrando características gerais do comportamento das doenças alérgicas do ambulatório. Isso possibilita a elaboração de planos de ação para melhorar e ampliar o serviço, possibilitando um diagnóstico mais rápido e tratamento adequado. Dessa forma, o presente estudo teve como objetivo avaliar o perfil dos pacientes registrados no ambulatório de Alergia e Imunologia do Hospital Universitário de Alagoas. 
Método

Estudo transversal analítico com dados secundários, realizado através da análise do livro de registro de diagnósticos do ambulatório de Alergia e Imunologia do Hospital Universitário de Alagoas. O livro é um registro que não identifica os pacientes, sendo anotado apenas sexo, idade e diagnóstico.

No período de outubro de 2016 a setembro de 2017 foram realizadas 1.164 consultas, 582 pacientes foram atendidos no ambulatório e 213 pacientes foram incluídos no livro de registro. São registrados os pacientes portadores de imunodeficiências primárias, alergia alimentar, reação medicamentosa, dermatite atópica grave, dermatite de contato grave, asma grave e anafilaxia. Neste estudo foram excluídos 8 pacientes por informações incompletas.

As variáveis analisadas foram idade, sexo e diagnóstico. O banco de dados foi montado no programa Microsoft Excel 2017 e analisado pelo programa EpiInfo 7.2.2.6. Os dados estão apresentados por meio de frequências simples e relativas. Para a análise estatística usou-se o teste de Quiquadrado no nível de confiança de 95\%. Para a análise da relação entre idade e alergia, os pacientes foram separados em 3 faixas etárias, de zero a menos de 10 anos, de 10 a 18 anos e maiores de 18 anos. As alergias foram separadas entre alimentares e não alimentares, e para a análise da relação entre idade e alergias alimentares, foram criados dois grupos: "APLV/leite" e “outras substâncias".

Foram seguidos os critérios éticos da resolução 466/12 do MS.

\section{Resultados}

Dos 205 pacientes registrados e que foram incluídos na amostra, 95 $(46,34 \%)$ eram do sexo feminino e 110 $(53,66 \%)$ do masculino. A idade média foi de 16,5 anos, com desvio padrão de 17,3 .

A alergia alimentar esteve presente no diagnóstico de $98(47,8 \%)$ pacientes, sendo a mais prevalente. $\mathrm{O}$ principal agente desencadeante da alergia alimentar foi o leite de vaca, sendo responsável por 50 (51,02\%), seguida do ovo 13 (13,3\%). Alguns alimentos como soja e pimenta apareceram apenas uma vez e foram alocadas no grupo de outros. A Tabela 01 apresenta a distribuição dos agentes desencadeantes da alergia alimentar. 
Tabela 01- Agentes Desencadeantes de Alergia Alimentar

\begin{tabular}{ccc}
\hline Desencadeante & $\mathrm{N}$ & $\%$ \\
\hline Leite de vaca & 50 & 51,00 \\
Ovo & 13 & 13,30 \\
Crustáceos & 12 & 12,24 \\
Frutas & 5 & 5,10 \\
Chocolate & 3 & 3,06 \\
Peixe & 2 & 2,04 \\
Trigo & 2 & 2,04 \\
Outros & 11 & 11,22 \\
Total: & 98 & 100,00 \\
\hline
\end{tabular}

Ao analisar a relação entre o grupo de idade com grupo de alergia (dicotômica alergia alimentar $\mathrm{x}$ não alimentar) e entre o grupo idade e alergia alimentar (dicotômica alergia a leite de vaca $\mathrm{x}$ outras substâncias) foi encontrado associação, com $\mathrm{p}=0,006$ e $\mathrm{p}=0,020$ respectivamente. (Tabelas $2 \mathrm{e}$ 3).
Ao observar a frequência de alergia alimentar e não alimentar por faixa etária, foi percebido que a alergia alimentar foi mais prevalente no grupo de 0 a menos de 10 anos, sendo 60 $(58,25 \%)$. No que concerne as alergias não alimentares, elas foram mais prevalentes na faixa etária juvenil e adulta, conforme demonstra a Tabela 02:

\section{Tabela 02 - Distribuição das Idades Segundo Alergia Alimentar}

\begin{tabular}{cccccc}
\hline & \multicolumn{2}{c}{ Alimentar } & \multicolumn{2}{c}{ Não Alimentar } & $\mathrm{p}$ \\
Grupos etários & $\mathrm{n}$ & $\%$ & $\mathrm{n}$ & $\%$ & \\
\hline De 0 a menos de 10 & 60 & 58,25 & 43 & 41,75 & \\
De 10 a 18 & 20 & 43,48 & 26 & 56,52 & 0,006 \\
Maior igual a 18 & 18 & 32,14 & 38 & 67,86 & \\
Total de pacientes & 98 & 47,80 & 107 & 52,20 & \\
\hline
\end{tabular}

Ao correlacionar, por faixa etária, a alergia alimentar mais comum com as demais alergias alimentares, foi encontrada maior prevalência da alergia 
ao leite de vaca no grupo de 0 a menos de 10 anos, correspondendo 34 $(56,67 \%)$. Os alimentos presentes no grupo "outras substancias" foram: ovo, crustáceos, frutas, chocolate, peixe, trigo, entre outros. Estes alimentos foram responsáveis pela maioria das alergias alimentares na faixa etária acima de 18 anos, encontrados em 12 $(66,66 \%)$ dos pacientes, como pode ser verificado na Tabela 03 .

Tabela 03 - Distribuição das Idades Segundo Alergia a Leite e Outras Substancias

\begin{tabular}{|c|c|c|c|c|c|}
\hline \multirow{2}{*}{ rupos etários } & \multicolumn{2}{|c|}{ Leite/APLV } & \multicolumn{2}{|c|}{ Outras Substâncias } & \multirow{2}{*}{$\mathrm{p}$} \\
\hline & $\mathrm{n}$ & $\%$ & $\mathrm{n}$ & $\%$ & \\
\hline De 0 a menos de 10 & 34 & 56,67 & 26 & 43,33 & \\
\hline De 10 a 18 & 10 & 50,00 & 10 & 50,00 & 0,020 \\
\hline Maior igual a 18 & 6 & 33,33 & 12 & 66,66 & \\
\hline Total de pacientes & 50 & 51,02 & 48 & 48,98 & \\
\hline
\end{tabular}

Os demais quadros alérgicosimunológicos na amostra corresponderam principalmente á reação á fármacos e as imunodeficiências primárias. As reações a fármacos foram diagnosticadas em 39,5\% dos pacientes, sendo composta principalmente pelos anti-inflamatórios não esteiroidais $77,7 \%$, seguida dos antibióticos 18,5\%. Alguns fármacos como a aminofilina $\mathrm{e}$ o valproato de sódio tiveram apenas uma ocorrência e foram agrupados no grupo outros 6,17\%. Dentre os AntiInflamatórios Não Esteroidais (AINES), os mais alergênicos foram: dipirona 63,5\%, ácido acetilsalicílico $15,8 \%$, diclofenaco 7,93\% e ibuprofeno 7,93\%. Em relação aos antibióticos, as
Penicilinas corresponderam a $80 \%$ e as Sulfonamidas a $20 \%$.

As imunodeficiências primárias foram identificadas em $5,4 \%$ da amostra. Destes, 4 36,3\% foram diagnosticados com imunodeficiência comum variável, $27,3 \%$ com angioedema hereditário, 9,1\% com neutropenia grave, 9,1\% com ataxia e telangectasia, 9,1\% com doença de Castelman e 9,1\% com Síndrome de Wiskott-Aldrich. Nesta condição, em relação ao sexo encontrou-se $63,7 \%$ no sexo masculino e $36,3 \%$ no feminino.

Em uma menor proporção foram atendidos no ambulatório outras afecções que representaram 7,31\%, sendo elas: dermatite atópica grave, 
angioedema, urticária e dermatite de contato, ainda há outros diagnósticos que foram encontrados em apenas um paciente, como anafilaxia a picada de abelha e outro, alergia ao pelo de coelho.

\section{Discussão}

As doenças alérgicas são uma das principais causas de cuidados médicos na infância e seu impacto é mais pronunciado em famílias de baixa renda (7). Nestas, incluem a alergia alimentar, alergia medicamentosa, imunodeficiências primárias, rinite, angioedema e algumas formas de asma (7). Estudos prévios, como o de Orosco et al ${ }^{(7)}$, afirmam que a alergia é a causa mais comum de sintomas crônicos na faixa etária infanto-juvenil e que há uma predominância no sexo masculino. Este achado corrobora os dados encontrados neste estudo, onde se conseguiu significância estatística na associação entre alergia e faixa etária, tendo-se maior prevalência de alergia na faixa etária entre 0 a 10 anos.

No presente trabalho a alergia alimentar foi o diagnóstico mais prevalente, sendo a alergia ao leite de vaca a principal responsável, seguida da alergia ao ovo. A alergia ao leite de vaca foi mais prevalente na faixa etária pediátrica, dados estes que corroboram o estudo de Coelho et al. ${ }^{(8)}$, que identificou alergia alimentar em 81,3\% nas crianças de 0 a 9 anos, sendo ao leite a mais frequente, seguida da alergia ao ovo. Também foi encontrado significância estatística na análise da associação entre alergia ao leite e a faixa etária de 0 a 10 anos. Os alimentos alergênicos mais prevalentes se modificam de acordo com a faixa etária, o que pode ser percebido quando se compara com o estudo de Yamano et al. Este encontrou os crustáceos como os principais causadores de alergia alimentar (53,9\%), enquanto que a APLV correspondeu a apenas $4 \%$. Entretanto, a média de idade no estudo de Yamano foi de 32 anos e no presente estudo foi de 16,5 anos.

A reação medicamentosa foi $o$ segundo diagnóstico mais frequente, coexistido com a alergia alimentar em muitos casos. Os anti-inflamatórios não esteroidais foram os principais fármacos desencadeantes, seguidos dos antibióticos, representados principalmente pelas penicilinas. Dentre os AINES, os mais alergênicos foram a dipirona e o ácido acetilsalicílico (AAS). Essa prevalência também foi relatada por Aun et al. ${ }^{(10)}$, em estudo realizado no hospital universitário da FMUSP, que demonstrou que os AINES 
foram os principais desencadeadores de reações anafiláticas medicamentosas, em pacientes com alergia prévia ${ }^{(10)}$.

Contudo, estudos internacionais, como o de Qayoom et al. ${ }^{(10)}$ e Warren et al. ${ }^{(12)}$ encontraram resultados discordantes, nos quais os antibióticos foram a principal causa de alergia medicamentosa e os AINES ocuparam a segunda posição ${ }^{(10 ; 12)}$. Este fato poderia ser explicado pela diferença das médias de idade dos estudos, visto que, segundo Muñoz $^{(3)}$ a idade não é um fator de risco independente para alergia medicamentosa, visto que há uma diferença de padrões terapêuticos segundo a faixa etária ${ }^{(3)}$.

No período entre outubro de 2016 e setembro de 2017, foram atendidos onze pacientes com imunodeficiências primárias (IDPs), a maioria representada por homens (7:4). Segundo Roxo-Junior ${ }^{(6)}$ há uma preponderância no sexo masculino $(5: 1)$, uma vez que algumas IDPs são ligadas ao cromossomo $\mathrm{X}^{(6)}$. A prevalência de imunodeficiência é de 1:2000 e a incidência de algumas doenças específicas é entre 1:66.000 e 1:75.000 para imunodeficiência comum variável e entre 1:10.000 e 1:50.000 para angioedema hereditário (10). O Hospital universitário estudado é o hospital público referência do estado de Alagoas para o diagnóstico e acompanhamento dos pacientes portadores de imunodeficiências primárias, sendo justificado a prevalência maior do que o esperado dentro do ambulatório.

\section{Conclusão}

O perfil dos pacientes registrados no ambulatório de alergia e imunologia do Hospital universitário estudado é semelhante com os descritos da literatura. É importante ressaltar que esse hospital é referência para o Sistema Único de Saúde - SUS no estado de Alagoas para o diagnóstico e acompanhamento dos pacientes portadores de imunodeficiências primárias.

\section{Referências}

1. Arruda KL, Melo JML. A epidemia de alergia: por que as alergias estão aumentando no Brasil e no mundo? Braz J Allergy Immunol. 2015; 3(1):16.

2. Dyer AA, Gupta R. Epidemiology of Childhood Food Allergy. Pediatria [periódico na internet] 2013 [citado 2017 jun. 25]; 42(6): 101-5. Disponível em:https://www.ncbi.nlm.nih.gov/pub $\mathrm{med} / 23718236$. 
3. Muñoz GMT. Alergia a medicamentos: Conceptos básicos y actitud a seguir por el pediatra. Pediatria [periódico na internet] 2013 [citado 2017 jun. 25]; 1(1): 1-24. Disponível em:http://www.aeped.es/si tes/default/files/documentos/1-alergia farmacos_0.pdf.

4. Arminda J, Soares E, Sarinho E, Lorente F, Gama J, Barata LT. Prevalence and clinical features of adverse food reactions in Portuguese children. Medicina [periódico na internet] 2017 [citado 2017 jun. 29]; 40(13): 2-10. Disponível em:https://www.ncbi.nlm.nih.gov/pmc/ articles/PMC5585952/.

5. Mauro G, Ribeiro M, Gomes AP, Batista RS, Salem R, Oliveira L, et al. Classificação das imunodeficiências congênitas. Medicina [periódico na internet] 2009 [citado 2017 set. 10]; 66(6): 152-7. Disponível em:http://www.moreirajr.com.br/revist as.asp? fase $=$ r003\&id_materia $=4046$.

6. Roxo JP. Imunodeficiências primárias: aspectos relevantes para $\mathrm{o}$ pneumologista. Pneumologista [periódico na internet] 2009 [citado 2017 abr. 20]; 35(10): 1008-17. Disponível em: http://www.scielo.br/sc ielo.php?script $=$ sci_arttext\&pid $=\mathrm{S} 180$ 6-37132009001000010.

7. Rodríguez-Orozco AR, Peñaloza MSH, Castro, HP. Perfil de consulta en niños alérgicos provenientes de familias de bajos ingresos. Pediatria (Cuba) [periódico na internet] 2007 [citado 2017 abr. 19]; 79(3): 1-7. Disponível em: http://scielo.sld.cu/ scielo.php?script $=$ sci_arttext\&pid $=\mathrm{S} 00$ 34-7531200700030000.
8. Coelho MAQ, Cruz VD, Duarte R, Marques PQ, Almeida EG. Alergia alimentar em pacientes do ambulatório de alergia da Unimontes. Braz J Allergy Immunol.2015; 3(4):134.

9. Yamano EYS, Lobato IS, Cunha MNF, Pinho ARM. Alergia alimentar: clínica epidemiologia dos pacientes do serviço de Alergia e Imunologia da UFPA. Braz J Allergy Immunol. 2015; 3(4):133.

10. Aun MV, Blanca M, Garro LS, Ribeiro MR, Kalil J, Motta AA, et al. Nonsteroidal Anti-Inflammatory Drugs are Major Causes of Drug-Induced Anaphylaxis. Medicina [periódico na internet] 2014 [citado 2017 jun. 15]; 2(4):414-20. Disponível em: https://www.ncbi.nlm.nih.gov/pubmed/ 25017529.

11. Qayoom S, Bisati S, Manzoor S, Sameem F, Khan K. Adverse Cutaneous Drug Reactions - A Clinicodemographic Study in a Tertiary Care Teaching Hospital of the Kashmir Valley, India. Medicina [periódico na internet] 2017 [citado 2017 jul. 4]; 18(4):228-33. Disponível em: http://www.ams.ac.ir/AIM/NEWPUB/ 15/18/4/007.pdf.

12. Warren AM, Malhi R, Jani Y, Corrigan C, Walker D, Kariyawasam $\mathrm{H}$, et al. Prevalence of reported drug allergy and its impact on Beta lactam use with financial and health implications. Arch Asthma Allergy Immunol. Medicina [periódico na internet] 2017 [citado 2017 jun. 8]; 1(1):28-5. Disponível em: https://doi.org/10.29328/journal.haard. 1001004. 